\title{
Protective effects of Lactococcus chungangensis CAU 28 on alcohol-metabolizing enzyme activity in rats
}

\author{
Maytiya Konkit, ${ }^{1}$ Kiyoung Kim, Jong-Hwa Kim, and Wonyong Kim ${ }^{2}$ \\ Department of Microbiology, Chung-Ang University College of Medicine, Seoul 06974, Republic of Korea
}

\begin{abstract}
In this study, we investigated the beneficial effects of Lactococcus chungangensis CAU 28, a bacterial strain of nondairy origin, on alcohol metabolism in rats treated with ethanol, focusing on alcohol elimination and prevention of damage and comparing the effects with those observed for Lactococcus lactis ssp. lactis ATCC 19435. Male Sprague-Dawley rats were orally administered $20 \%$ ethanol and 3 substrates (freeze-dried cells, cream cheese, and yogurt) containing Lc. chungangensis CAU 28 or Lc. lactis ssp. lactis ATCC 19435, which were provided $1 \mathrm{~h}$ before or $1 \mathrm{~h}$ after ethanol ingestion. Blood samples were collected from the tail veins of the rats at $1,3,6,12$, and $24 \mathrm{~h}$ after ingestion of ethanol, Lc. chungangensis CAU 28 substrate, or Lc. lactis ssp. lactis ATCC 19435 substrate. Alcohol and acetaldehyde concentrations in the Lc. chungangensis CAU 28 substrate-treated rats were significantly reduced in a time-dependent manner compared with those in the $L c$. lactis ssp. lactis ATCC 19435 substrate-treated rats. Among the experimental groups, treatment with cream cheese before ingestion of $20 \%$ ethanol was found to be the most effective method for reducing both alcohol and acetaldehyde levels in the blood. Alanine aminotransferase and aspartate aminotransferase activities in the Lc. chungangensis CAU 28 substrate-treated rats were significantly lower than those in the positive controls. Moreover, in the Lc. chungangensis CAU 28 cream cheese-treated group, rats showed a reduction of liver enzymes by up to $60 \%$, with good effectiveness observed for both pre- and post-ethanol ingestion. These results suggested that intake of lactic acid bacteria, particularly in Lc. chungangensis CAU 28-supplemented dairy products, may reduce blood alcohol and acetaldehyde concentrations, thereby mitigating acute
\end{abstract}

Received October 15, 2017.

Accepted March 12, 2018.

${ }^{1}$ Current address: Department of Biology, Faculty of Science, Chiang Mai University, Chiang Mai 50200, Thailand.

${ }^{2}$ Corresponding author: kimwy@cau.ac.kr alcohol-induced hepatotoxicity by altering alcoholmetabolizing enzyme activities.

Key words: Lactococcus, alcohol dehydrogenase, aldehyde dehydrogenase, aspartate aminotransferase, alanine aminotransferase

\section{INTRODUCTION}

Alcoholic beverages have been consumed by humans since the beginning of recorded history (Room et al., 2005). Fermented drinks are now prepared and consumed globally, affecting the cultural acceptance of alcohol (Room et al., 2002). However, problems associated with alcohol consumption have increased in the last $30 \mathrm{yr}$, with substantial advances in our understanding of drinking problems as well as their prevention and treatment (Room et al., 2005). After consumption of alcoholic beverages, alcohol is absorbed into the blood and diluted by the total body water. The oxidation of alcohol occurs primarily in the liver, and the major pathway for alcohol metabolism involves the production of alcohol dehydrogenase (ADH), which converts alcohol into acetaldehyde through enzymatic oxidation. Acetaldehyde is an organic chemical compound that is highly toxic to the body, even at low concentrations. However, aldehyde dehydrogenase (ALDH) rapidly oxidizes acetaldehyde to acetate (Maher, 1997; Vanni et al., 2000). Pearl (1926) reported that excessive alcohol consumption could lead to death. Moreover, alcohol consumption contributes to the incidence of several diseases, such as breast cancer, coronary heart disease, stroke, diabetes, and liver injury (Rehm et al., 1997; Smith-Warner et al., 1998; Corrao et al., 2000).

Various lactic acid bacteria (LAB) are currently being used to prevent and treat diseases. The most commonly used species include Lactobacillus acidophilus, Lactobacillus bifidus, Lactobacillus bulgaricus, Lactobacillus casei, Lactobacillus rhamnosus, Lactobacillus plantarum, Lactobacillus fermentum, Streptococcus thermophilus, and Enterococcus faecium. Lactic acid bacteria benefit the host when administered at the proper amounts (Kalliomäki et al., 2001; Brown and Valiere, 2004). Additionally, probiotics can be beneficial to human health 
by affecting gastrointestinal infections, antimicrobial activity, lactose metabolism, serum cholesterol levels, immune system function, and because of their antimutagenic, anticarcinogenic, and antidiarrheal properties (Gomes and Malcata, 1999; Agerholm-Larsen et al., 2000; Gotcheva et al., 2002; Nomoto, 2005; Imase et al., 2007; Shah, 2007).

Some oral streptococci have been shown to exhibit $\mathrm{ADH}$ activity and produce acetaldehyde in vitro during ethanol incubation. In particular, Streptococcus salivarius, Streptococcus intermedius, and Streptococcus mitis produce high amounts of acetaldehyde in response to exposure to 11 or $1,100 \mathrm{~m} M$ ethanol (Kurkivuori et al., 2007). Leuconostoc cremoris also shows NADPdependent alcohol dehydrogenase activity for reducing acetaldehyde to ethanol (Lees and Jago, 1976). The thermophilic bacterium Thermoanaerobium brockii contains both NADP-linked ADH and NAD-linked $\mathrm{ADH}$ and has thus attracted considerable interest for industrial alcohol and enzyme production (Lamed and Zeikus, 1981). In addition, LAB of the genus Lactobacillus, including Lactobacillus GG ATCC 53103, can metabolize ethanol and acetaldehyde in vitro, as determined by gas chromatography of the bacterial suspension after incubating the culture for $1 \mathrm{~h}$ with $22 \mathrm{mM}$ ethanol (Nosova et al., 2000).

Another LAB, Lactococcus chungangensis CAU 28, has been shown to express several genes that are relevant for dairy milk manufacturing, such as $S$-adenosylmethionine (Konkit et al., 2014). In addition, this strain has been shown to produce ADH and ALDH to reduce alcohol and acetaldehyde levels in vitro and in vivo (Konkit et al., 2015, 2016). A recent study showed that this strain could reduce atopic dermatitis symptoms in mice (Choi et al., 2016).

Accordingly, in this study, the $\mathrm{ADH}$ and ALDH activities of $L c$. chungangensis CAU 28 were characterized and compared with those of Lactococcus lactis ssp. lactis ATCC 19435 through administration in freezedried cells, cream cheese, or yogurt $1 \mathrm{~h}$ before or after administration of ethanol. Thus, Lc. chungangensis CAU 28 may have applications as an effective alcohol detoxicant.

\section{MATERIALS AND METHODS}

\section{Bacterial Strain Culture and Sample Preparation}

Lactococcus chungangensis CAU 28 and Lc. lactis ssp. lactis ATCC 19435 (hereafter, Lc. chungangensis and Lc. lactis) were cultured in tryptic soy broth (Becton, Dickinson and Co., Sparks, NV) at $30^{\circ} \mathrm{C}$ for $24 \mathrm{~h}$.

To evaluate alcohol metabolism, freeze-dried cells, cream cheese, and yogurt were prepared with Lc. chun- gangensis and Lc. lactis. For freeze-dried cell preparation, cells cultured in tryptic soy broth at $30^{\circ} \mathrm{C}$ for $24 \mathrm{~h}$ were harvested by centrifugation at $12,000 \times g$ and $4^{\circ} \mathrm{C}$ for $20 \mathrm{~min}$ and then dried using a freeze dryer (Hanil Science Inc., Gimpo, Korea). Cream cheese was made as described previously (Konkit et al., 2015). Briefly, pasteurized milk (Pasteur Milk Co., Seoul, Korea) was heated at $68^{\circ} \mathrm{C}$ for $30 \mathrm{~min}$, cooled, and then $5 \%$ ( $\mathrm{vol} / \mathrm{vol}$ ) cell culture of Lc. chungangensis or Lc. lactis was added and the milk incubated at $30^{\circ} \mathrm{C}$ for $48 \mathrm{~h}$. The milk was acidified during this period, stirred, and heated at $70^{\circ} \mathrm{C}$ for $5 \mathrm{~min}$, and the whey was separated using a cloth bag. Curd was set, and the whey was drained by adding $0.5 \%$ salt. Yogurt was prepared according to a method described previously (Konkit et al., 2015). Briefly, pasteurized milk was heated at $93^{\circ} \mathrm{C}$, stirred gently, and then cooled until it was warm to touch, around 44 to $46^{\circ} \mathrm{C}$, after which the milk was occasionally stirred to prevent skin formation. A cup of the warm milk was scooped out, the inoculum strain $(1 \% \mathrm{vol} / \mathrm{vol})$ was added to it, and the yogurt was kept to set overnight. Finally, both cream cheese and yogurt samples were freeze-dried and stored in the dark at $4^{\circ} \mathrm{C}$ until further use.

\section{Administration of Substrate to Animals}

Five-week-old male Sprague Dawley rats were purchased from Samtako (Osan, Korea). Rats were acclimated to cages at $22^{\circ} \mathrm{C}$ and a 12 -h day/night cycle, and these conditions were maintained throughout the experiments, beginning $1 \mathrm{wk}$ before the start of the experiments. The rats were divided into 8 groups $(\mathrm{n}=$ 3 for each group): (1) negative control group (water administration); (2) positive control group (20\% ethanol administration); (3) group administered Lc. chungangensis freeze-dried cells $\left(10^{9} \mathrm{cfu} / \mathrm{mL}\right)$; (4) group administered cream cheese made with Lc. chungangensis (1.4 $\mathrm{g} / \mathrm{kg}) ;(5)$ group administered yogurt made from $L c$. chungangensis $(1.4 \mathrm{~g} / \mathrm{kg})$; (6) group administered $L c$. lactis freeze-dried cells $\left(10^{9} \mathrm{cfu} / \mathrm{mL}\right) ;(7)$ group administered cream cheese made from Lc. lactis $(1.4 \mathrm{~g} / \mathrm{kg})$; and (8) group administered yogurt made from Lc. lactis $(1.4 \mathrm{~g} / \mathrm{kg})$. All rats except those in the negative group were administered the substrate orally and groups were either given $20 \%$ ethanol first followed by substrate or substrate first and then $20 \%$ ethanol (Figure 1).

\section{Determination of Alcohol and Acetaldehyde Concentrations in Rat Blood}

Whole-blood samples were collected from the tail veins of Sprague Dawley rats in serum separation tubes at $1,3,6,12$, and $24 \mathrm{~h}$ after ethanol and substrate 
administration. The blood samples were centrifuged at $12,000 \times g$ for $15 \mathrm{~min}$ and incubated at $4^{\circ} \mathrm{C}$ for 10 min. The supernatant (comprising blood serum) was used for analyses of alcohol and aldehyde concentrations. The reaction mixture, containing $0.3 \mathrm{M} \mathrm{KH}_{2} \mathrm{PO}_{4}$ buffer (pH 9.0), $49 \mathrm{mM} \mathrm{NAD}^{+}$, and serum in a final volume of $3 \mathrm{~mL}$, was incubated at $20^{\circ} \mathrm{C}$ for $5 \mathrm{~min}$, and its absorbance (A1) was measured at $340 \mathrm{~nm}$ using a spectrophotometer (Tecan, Männedorf, Switzerland). After measurement, $50 \mu \mathrm{L}$ of $\mathrm{ADH}$ for alcohol analysis or $50 \mu \mathrm{L}$ of $\mathrm{ALDH}$ for aldehyde analysis was added to the mixture, and the mixture was incubated at $20^{\circ} \mathrm{C}$ for $5 \mathrm{~min}$. The absorbance (A2) was measured at $340 \mathrm{~nm}$ using the Infinite 200 NanoQuant spectrophotometer (Tecan). Blood alcohol and aldehyde concentrations were calculated using the following equations:

Alcohol concentration $=0.7259 / 3.6 \times \Delta \mathrm{A}$,

Aldehyde concentration $=0.7158 / 3.6 \times \Delta \mathrm{A}$,

where $\Delta \mathrm{A}=$ sample $(\mathrm{A} 2-\mathrm{A} 1)-$ blank $(\mathrm{A} 2-\mathrm{A} 1)$.

\section{Determination of Aspartate Aminotransferase and Alanine Aminotransferase in Rat Blood}

All rats were fasted for $12 \mathrm{~h}$ before blood collection. Whole-blood samples were collected from the hearts of the experimental Sprague Dawley rats and analyzed for total aspartate aminotransferase (AST) and alanine aminotransferase (ALT) at Green Cross Biotherapeutics Inc. (Yongin, South Korea).

\section{Statistical Analysis}

All results are presented as means \pm standard deviations. One-way ANOVA with Tukey's post hoc test was applied using the predictive analytic software statistics package for Windows (SPSS Inc./IBM Corp., Armonk, NY). Differences with $P$-values $<0.05$ were considered statistically significant.

\section{RESULTS}

\section{ADH Activity in Rats Treated with $20 \%$ Ethanol First and then Administered Substrate}

In vivo studies were conducted to measure alcohol concentrations in rat sera after administration of $20 \%$ ethanol, followed by administration of Lc. chungangensis or Lc. lactis substrates after $1 \mathrm{~h}$. From 1 to $3 \mathrm{~h}$, the blood alcohol concentration of the positive group was significantly increased $(29.64-30.55 \mathrm{mg} / \mathrm{dL} ; P$ $=0.01$ ) compared with those of the treated sample groups (Figure 2A and 2B). At 6, 12, and $24 \mathrm{~h}$, we observed differences in blood alcohol levels between treated groups administered freeze-dried cells $(P=$ $0.01)$, cream cheese $(P=0.03)$, and yogurt $(P=0.01)$ and compared with the positive control group $(P=$ 0.01). The blood alcohol concentrations in the rats treated with freeze-dried cells, cream cheese, and yogurt were $20.25,20.34$, and $19.64 \mathrm{mg} / \mathrm{dL}$, respectively, for Lc. chungangensis and 22.57, 21.45, and $20.79 \mathrm{mg} /$ $\mathrm{dL}$, respectively, for Lc. lactis, with a positive control of $22.61 \mathrm{mg} / \mathrm{dL}$ at $6 \mathrm{~h} ; 19.37,19.23$, and $19.13 \mathrm{mg} / \mathrm{dL}$, re-

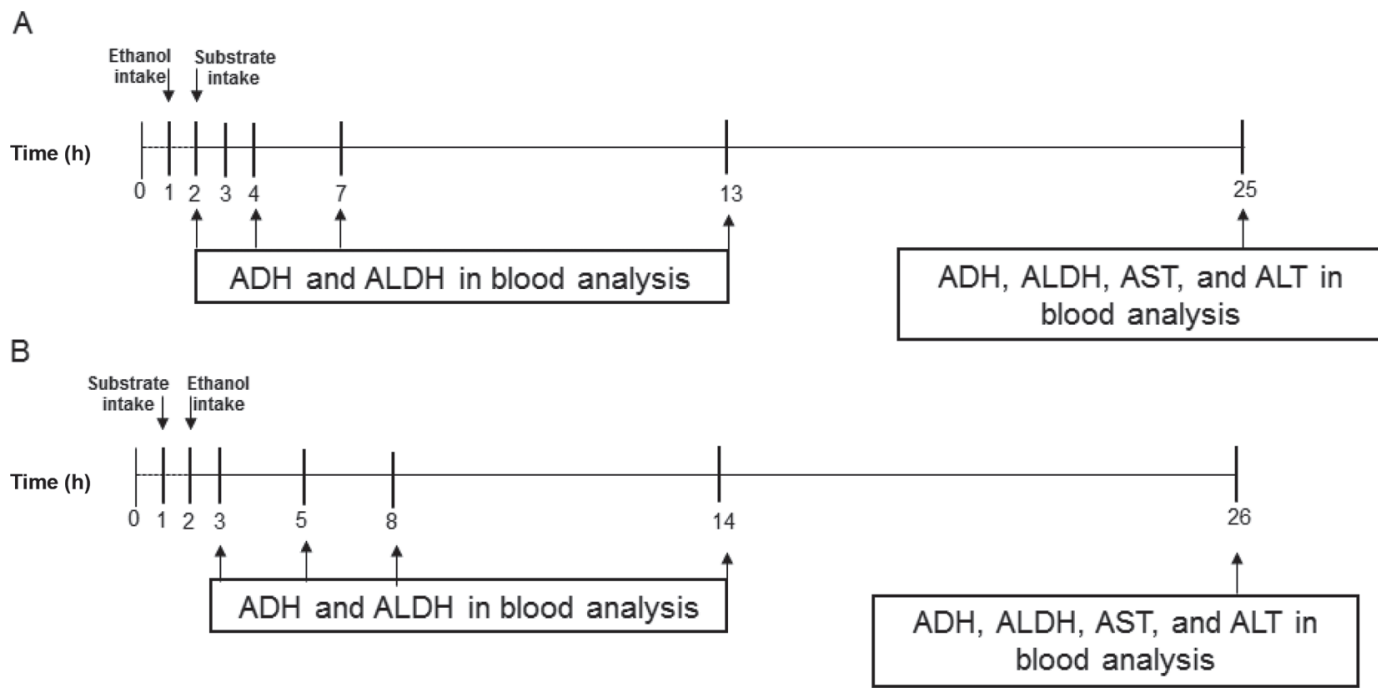

Figure 1. Schematic of the experiment schedule. (A) Treatment of rats with $20 \%$ ethanol first and then with Lactococcus chungangensis CAU 28 and Lactococcus lactis ssp. lactis ATCC 19435 in substrates (freeze-dried cells, cream cheese, and yogurt). (B) Administration of Lc. chungangensis CAU 28 and Lc. lactis ssp. lactis ATCC 19435 substrates first and then $20 \%$ ethanol. ADH = alcohol dehydrogenase; ALDH = aldehyde dehydrogenase; AST = aspartate aminotransferase; ALT = alanine aminotransferase. 

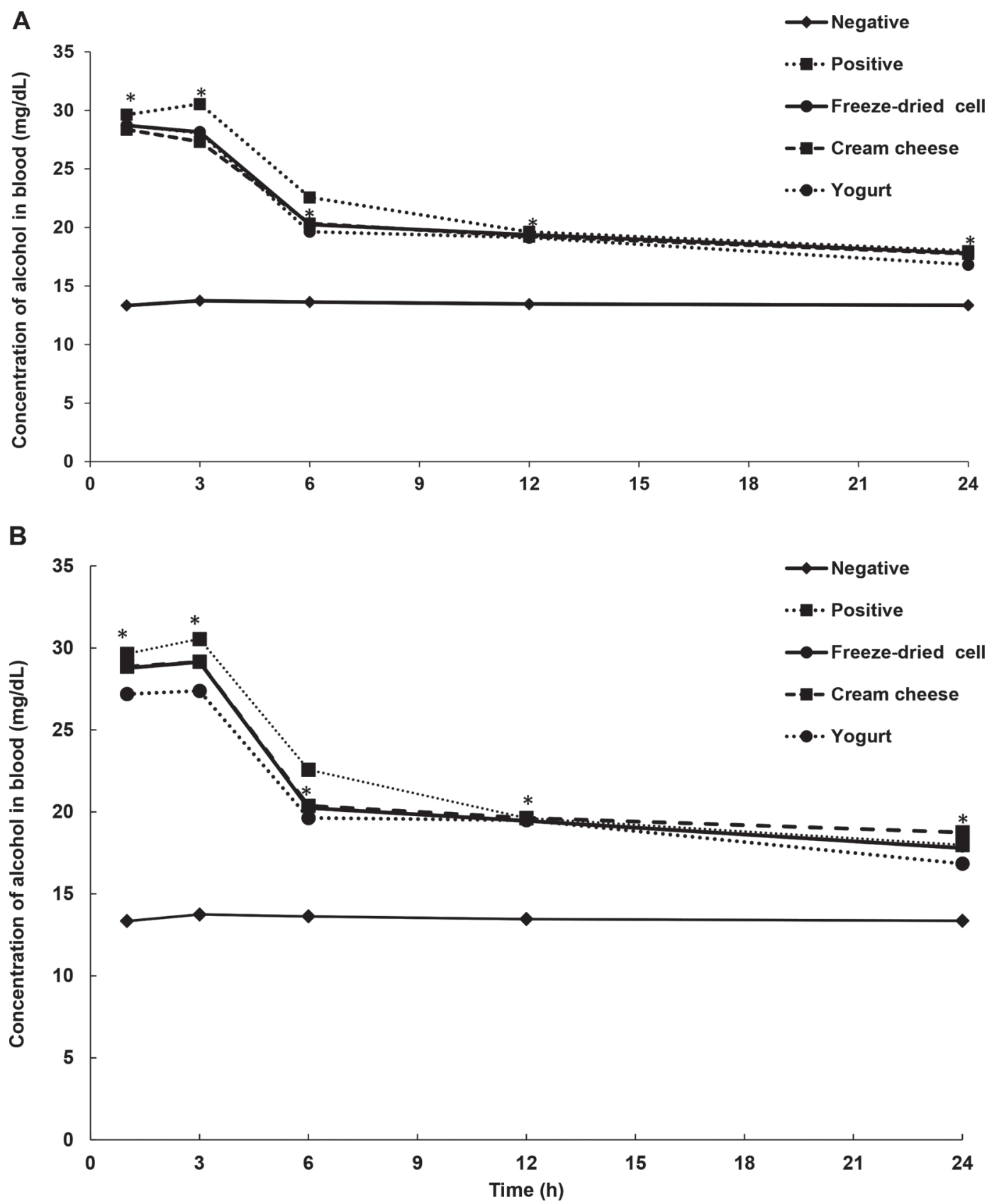

Figure 2. Alcohol dehydrogenase concentrations in rat blood after treatment with $20 \%$ ethanol first and then substrates. (A) Lactococcus chungangensis CAU 28 in 1 of 3 substrates: freeze-dried cells, cream cheese, or yogurt; (B) Lactococcus lactis ssp. lactis ATCC 19435 in substrates. Negative $=$ water only, Positive $=20 \%$ ethanol only. Results are expressed as means \pm SD of 3 independent experiments; the 3 values of alcohol dehydrogenase are very close, so error bars are not clearly visible. ${ }^{*} P<0.05$.

spectively, for Lc. chungangensis and 21.68, 20.67, and $20.79 \mathrm{mg} / \mathrm{dL}$, respectively, for Lc. lactis, with a positive control of $22.07 \mathrm{mg} / \mathrm{dL}$ at $12 \mathrm{~h}$; and 17.81, 17.74, and $16.82 \mathrm{mg} / \mathrm{dL}$, respectively, for Lc. chungangensis and $20.67,19.75$, and $17.83 \mathrm{mg} / \mathrm{dL}$, respectively, for $L c$. lactis, with a positive control of $19.33 \mathrm{mg} / \mathrm{dL}$ at $24 \mathrm{~h}$. Interestingly, at $24 \mathrm{~h}$, the blood alcohol concentrations in rats from the yogurt group of Lc. chungangensis were lower than those of the other treated groups and the positive control $(P=0.03)$.

\section{ADH Activity in Rats Administered Substrate First and then Treated with $20 \%$ Ethanol}

Alcohol concentrations in rat serum were measured after administration of Lc. chungangensis and Lc. lactis 
substrates, followed by treatment with $20 \%$ ethanol after $1 \mathrm{~h}$. At $1 \mathrm{~h}$ after administration of $20 \%$ ethanol, the blood alcohol concentration of the positive group was significantly increased $(29.54 \mathrm{mg} / \mathrm{dL} ; P=0.01)$ compared with those of the other treated groups (Figure 3A and $3 \mathrm{~B}$ ). Interestingly, rats administered cream cheese with Lc. chungangensis or Lc. lactis had the lowest blood alcohol concentrations (23.05 and $25.2 \mathrm{mg} / \mathrm{dL}$, respectively; $P=0.01$ ) among all groups. From 3 to 6 $\mathrm{h}$, rats in groups administered freeze-dried cells, cream cheese, and yogurt had lower blood alcohol concentrations than those in the positive group. At $12 \mathrm{~h}$, rats in the group treated with Lc. chungangensis and Lc. lactis cream cheese had the lowest blood alcohol concentrations of 16.26 and $19.35 \mathrm{mg} / \mathrm{dL}$, respectively $(P=0.01)$, compared with those of the other treated groups. In addition, rats in groups administered freeze-dried cells and yogurt also had low blood alcohol concentrations of 17.73 and $17.74 \mathrm{mg} / \mathrm{dL}$, respectively, in Lc. chungangensis, and 21.37 and $20.83 \mathrm{mg} / \mathrm{dL}$, respectively, in Lc. lactis; however, there was a significant difference between groups. At $24 \mathrm{~h}$, we observed differences in blood alcohol levels between treated groups administered freeze-dried cells $(P=0.01)$, cream cheese $(P=$ $0.01)$, and yogurt $(P=0.01)$, and the positive control group $(P=0.01)$. Moreover, the blood alcohol concentrations of the Lc. chungangensis substrate groups were $16.30,16.14$, and $17.48 \mathrm{mg} / \mathrm{dL}$, respectively, and those in the Lc. lactis substrate groups were 20.09, 18.19, and $19.58 \mathrm{mg} / \mathrm{dL}$, respectively. Notably, at $24 \mathrm{~h}$, the blood alcohol concentration in rats administered cream cheese from Lc. chungangensis was the lowest among all treated groups, Lc. lactis-treated groups, and the positive control $(P=0.01)$.

\section{ALDH Activity in Rats Treated with $20 \%$ Ethanol First and then Administered Substrate}

From 1 to $3 \mathrm{~h}$, blood acetaldehyde concentrations in the positive group were significantly increased (27.94-29.22 mg/dL; $P=0.01)$ compared with those of the other treated groups (Figure $4 \mathrm{~A}$ and $4 \mathrm{~B}$ ). At 6 $\mathrm{h}$, we observed differences in blood acetaldehyde levels between treated groups administered freeze-dried cells $(P=0.01)$, cream cheese $(P=0.03)$, and yogurt $(P=$ $0.01)$ and the positive control group $(P=0.01)$. Furthermore, blood acetaldehyde concentrations in the $L c$. chungangensis substrate groups were 20.34, 18.58, and $17.93 \mathrm{mg} / \mathrm{dL}$, respectively, and those in the Lc. lactis substrate groups were $21.72,19.65$, and $19.40 \mathrm{mg} / \mathrm{dL}$, respectively. Even at 12 and $24 \mathrm{~h}$, there were differences in blood acetaldehyde concentrations between rats in treated groups administered freeze-dried cells $(P=$ $0.01)$, cream cheese $(P=0.03)$, and yogurt $(P=0.01)$ and in the positive control group $(P=0.01)$. The blood acetaldehyde concentrations of the groups treated with Lc. chungangensis at $24 \mathrm{~h}$ were 17.47, 16.84, and 16.67 $\mathrm{mg} / \mathrm{dL}$, respectively, whereas those of the Lc. lactis substrate groups were $17.90,17.74$, and $17.66 \mathrm{mg} / \mathrm{dL}$, respectively. In addition, from 1 to $24 \mathrm{~h}$, the blood acetaldehyde concentrations in rats fed yogurt made with Lc. chungangensis were significantly reduced compared with those of the other treated groups, the Lc. lactistreated groups, and the positive control $(P=0.03)$.

\section{ALDH in Rats Administered Substrate First and then Treated with $20 \%$ Ethanol}

After $1 \mathrm{~h}$, blood acetaldehyde concentrations in rats in the positive group were significantly increased (32.69 $\mathrm{mg} / \mathrm{dL} ; P=0.01)$ compared with those in rats from other treated groups (Figure 5A and 5B). At 3, 6, 12, and $24 \mathrm{~h}$, we observed differences in blood alcohol levels between treated groups administered freeze-dried cells $(P=0.01)$, cream cheese, $(P=0.01)$, and yogurt $(P=$ $0.01)$ and the positive control group $(P=0.01)$. The blood acetaldehyde concentrations in the Lc. chungangensis substrate groups were 19.25 to $19.64,15.91$ to 17.23 , and 17.33 to $18.53 \mathrm{mg} / \mathrm{dL}$, respectively, and those in the Lc. lactis substrate groups were 22.96 to 21.29 , 18.9 to 17.2 , and 19.57 to $18.41 \mathrm{mg} / \mathrm{dL}$, respectively, at 3 to $6 \mathrm{~h}$. The blood acetaldehyde concentrations were $16.72,15.36$, and $15.43 \mathrm{mg} / \mathrm{dL}$, respectively, in the $L c$. chungangensis substrate groups and $17.18,16.76$, and $16.92 \mathrm{mg} / \mathrm{dL}$, respectively, in the Lc. lactis substrate groups at $24 \mathrm{~h}$. Additionally, from 1 to $24 \mathrm{~h}$, the blood acetaldehyde concentrations in rats administered cream cheese from Lc. chungangensis were the lowest among all treated groups, the Lc. lactis-treated groups, and the positive control $(P=0.01)$.

\section{Blood AST and ALT Levels in Rats Treated with $20 \%$ Ethanol and Administered Substrates}

Aspartate aminotransferase and ALT are enzymes used as blood biomarkers to assess alcohol abuse and liver injury. In this experiment, we found that blood AST levels in rats from the positive group were highest (67 U/L following treatment with $20 \%$ alcohol first, and $82.67 \mathrm{U} / \mathrm{L}$ following administration of substrates first; $P=0.01$ ) among all groups (Figure $6 \mathrm{~A}$ and $7 \mathrm{~A}$ ). Interestingly, rats in groups administered cream cheese and yogurt with Lc. chungangensis had significantly lower blood AST levels compared with those in the $L c$. lactis groups (35 and $40.67 \mathrm{U} / \mathrm{L}$, respectively, for $L c$. chungangensis, and 47.30 and $48.30 \mathrm{U} / \mathrm{L}$, respectively, for Lc. lactis when rats were treated with ethanol first; 42 and $43 \mathrm{U} / \mathrm{L}$, respectively, for Lc. chungangensis, and 

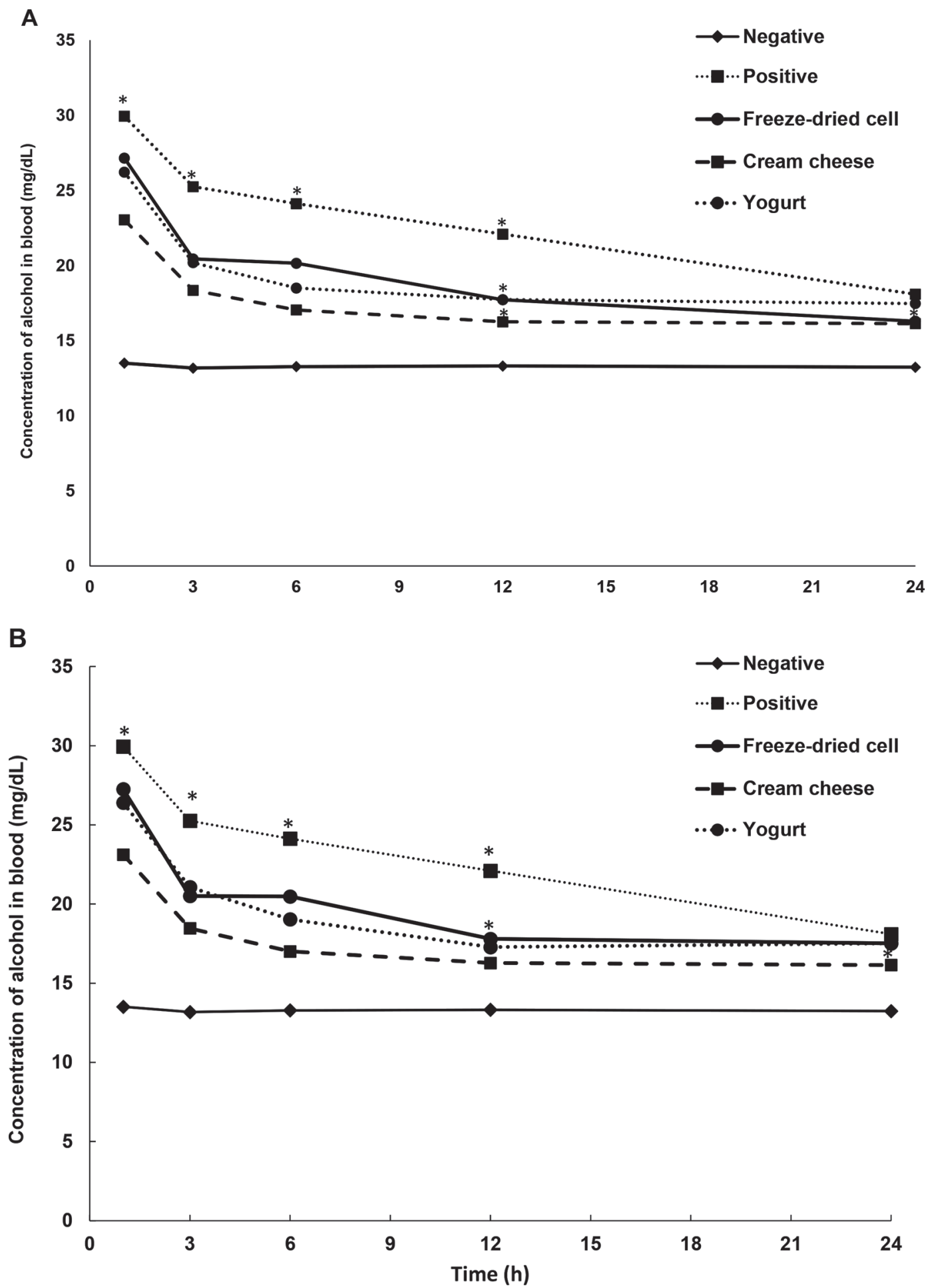

Figure 3. Alcohol dehydrogenase concentrations in rat blood after administration of substrates first and then $20 \%$ ethanol. (A) Lactococcus chungangensis CAU 28 in 1 of 3 substrates: freeze-dried cells, cream cheese, or yogurt; (B) Lactococcus lactis ssp. lactis ATCC 19435 substrates. Negative $=$ water only, Positive $=20 \%$ ethanol only. Results are expressed as means \pm SD from 3 independent experiments; the 3 values of alcohol dehydrogenase are very close, so error bars are not clearly visible. ${ }^{*} P<0.05$. 
41.30 and $44.30 \mathrm{U} / \mathrm{L}$, respectively, for Lc. lactis when the substrates were administered first; $P=0.01$ ).

Furthermore, blood ALT levels in rats from the positive group were $282.33 \mathrm{U} / \mathrm{L}$ following treatment with $20 \%$ alcohol first and were $310 \mathrm{U} / \mathrm{L}$ following administration of Lc. chungangensis and Lc. lactis first $(P$ $=0.01 ;$ Figure $6 \mathrm{~B}$ and $7 \mathrm{~B})$. Cream cheese and yogurt from Lc. chungangensis were found to reduce blood ALT levels in rats treated with $20 \%$ alcohol first or when administered substrates first, and ALT levels were significantly lower compared with those of positive and freeze-dried cell groups $(P=0.01)$.

\section{DISCUSSION}

Our previous studies have demonstrated that treatment with Lc. chungangensis CAU 28 cell culture and cream cheese prepared using this microorganism could reduce both alcohol and acetaldehyde levels in mouse blood (Konkit et al., 2015, 2016). Alcohol dependence
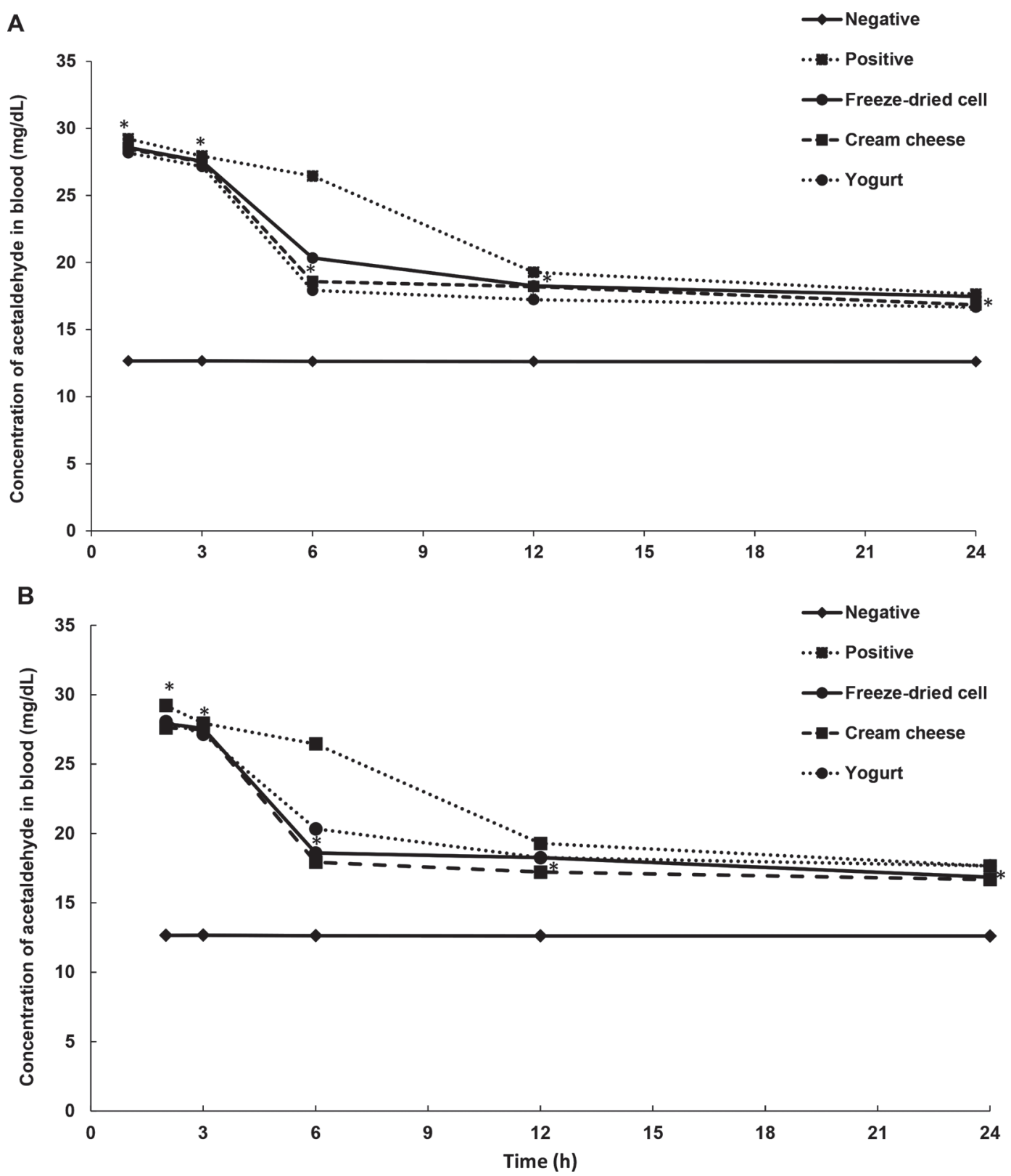

Figure 4. Acetaldehyde dehydrogenase concentrations in rat blood after treatment with $20 \%$ ethanol first and then substrates. (A) Lactococcus chungangensis CAU 28 in 1 of 3 substrates: freeze-dried cells, cream cheese, or yogurt; (B) Lactococcus lactis ssp. lactis ATCC 19435 substrates. Negative $=$ water only, Positive $=20 \%$ ethanol only. Results are expressed as means \pm SD from 3 independent experiments; the 3 values of alcohol dehydrogenase are very close, so error bars are not clearly visible. ${ }^{*} P<0.05$. 

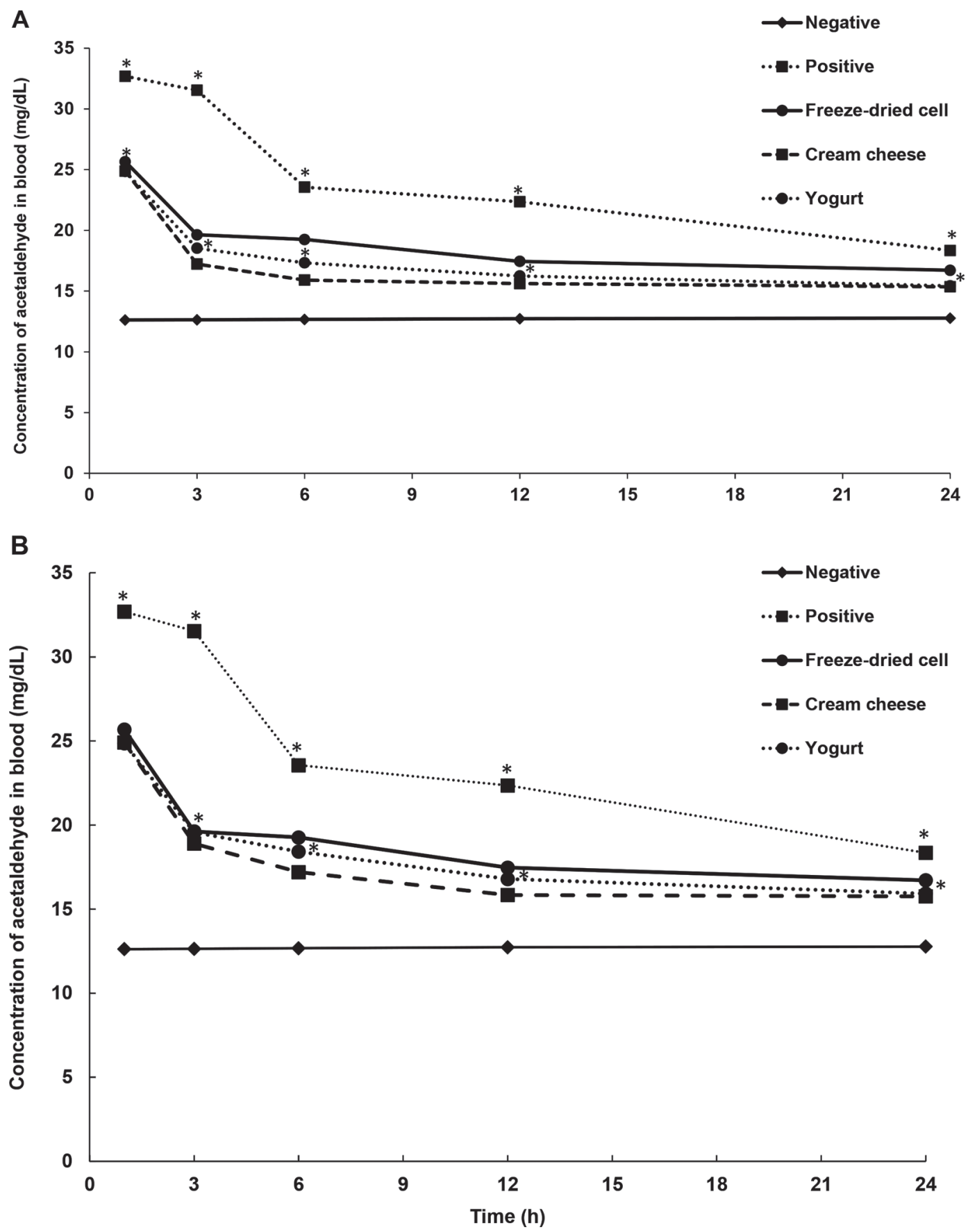

Figure 5. Acetaldehyde dehydrogenase concentrations in rat blood after administration of substrates first and then 20\% ethanol. (A) Lactococcus chungangensis CAU 28 in 1 of 3 substrates: freeze-dried cells, cream cheese, or yogurt; (B) Lactococcus lactis ssp. lactis ATCC 19435 substrates. Negative $=$ water only, Positive $=20 \%$ ethanol only. Results are expressed as means \pm SD from 3 independent experiments; the 3 values of alcohol dehydrogenase are very close, so error bars are not clearly visible. ${ }^{*} P<0.05$.

and alcohol abuse are the primary causes of end-stage liver disease and long-term morbidity and mortality (Sehrawat and Sultana, 2006). According to studies, alcoholic liver disease (ALD) has increased in several countries showing increased alcoholic beverage consumption (Takada et al., 1995; Rehm et al., 2003).
Alcoholic liver disease is a chronic disease related to social behavior and genetic polymorphisms in various enzymes and metabolic pathways that balance ethanol metabolism (Segawa et al., 2008). It accounts for $40 \%$ of deaths due to cirrhosis and $28 \%$ of all liver disease-related deaths in the United States (Voigt, 
2005). Alcohol abuse not only plays a role in ALD and hepatocellular carcinoma but also affects the brain and cardiovascular system (Setshedi et al., 2010). The most broadly recognized forms of ALD are alcoholic fatty liver disease (steatosis), acute alcoholic hepatitis, and alcoholic cirrhosis (Walsh and Alexander, 2000).
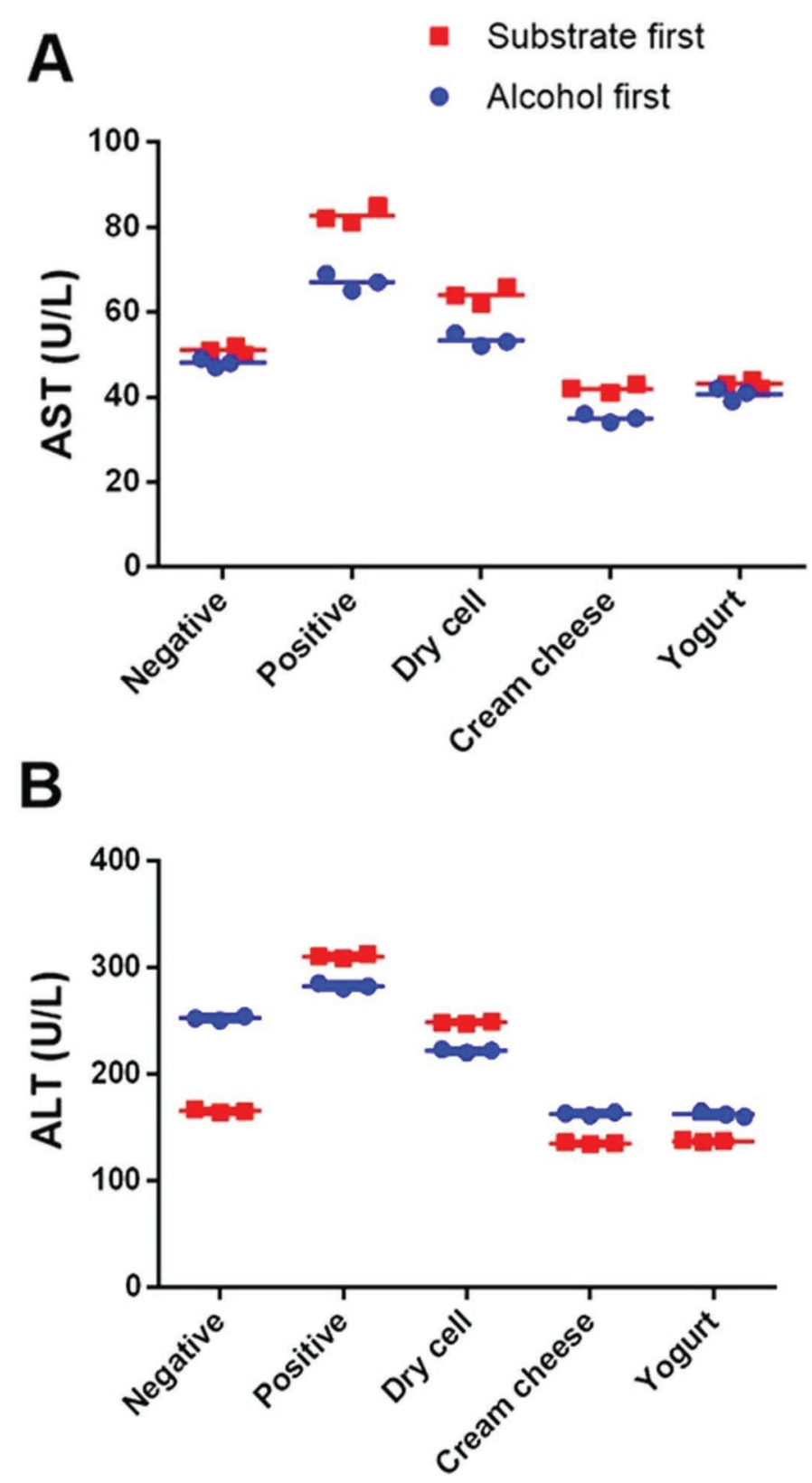

Figure 6. (A) Aspartate aminotransferase (AST), and (B) alanine aminotransferase (ALT) levels (in triplicate) in rat blood after treatment with $20 \%$ ethanol first and then Lactococcus chungangensis CAU 28 in 1 of 3 substrates: freeze-dried cells, cream cheese, or yogurt; or after administration of Lc. chungangensis CAU 28 in substrates first and then $20 \%$ ethanol. Negative $=$ water only, Positive $=20 \%$ ethanol only. Data are shown as the mean \pm SEM. Color version available online.
The gut microflora plays an important role in the pathogenesis of ALD. Nanji et al. (1994) reported that feeding live L. rhamnosus GG reduces endotoxemia and alcohol-induced liver injury in rats continuously infused with a diet of ethanol-containing liquid through an implanted gastric tube. Probiotics are microorganisms that usually have a human or animal origin (Schrezenmeir and de Vrese, 2001). Lactic acid bacteria with probiotic properties are generally enteric flora and play an important role in the human gastrointestinal tract ecosystem. Probiotic activities can have nutritional, physiological, and antimicrobial effects. Moreover, LAB are also potential adjuvants, and their oral administration activates both mucosal and systemic immune responses (Gerritse et al., 1990). Lactococcus species are LAB that can be developed into probiotics because of their benefits to the food industry. Additionally, Lactococcus species have been given "generally recognized as safe" status, similar to Lactobacillus species (Kimoto et al., 1999).

In this study, consumption of Lc. chungangensis CAU 28 substrates, such as freeze-dried cells, cream cheese, and yogurt, was found to significantly improve serum biochemical indices and influence alcohol-metabolizing enzymes in the liver, when administered before or after ethanol. Notably, blood alcohol and acetaldehyde levels were lower when rats were treated first with $20 \%$ ethanol and then with yogurt made from Lc. chungangensis than when rats were administered other Lc. chungangensis substrates or Lc. lactis substrates or were not administered LAB (positive control). Moreover, when rats were administered substrate first and then $20 \%$ ethanol, blood alcohol and acetaldehyde concentrations in rats were significantly decreased, particularly in the cream cheese group. When comparing the 2 experimental groups; that is, treatment with ethanol first and administration of substrates first, we found that blood alcohol and acetaldehyde levels in rats administered substrates first followed by $20 \%$ ethanol were lowest among the freeze-dried cell, cream cheese, and yogurt groups. These findings implied that administration of Lc. chungangensis substrates before alcohol consumption would be better for reducing blood alcohol and acetaldehyde levels.

Rogers et al. (1987) reported that food slows the absorption of alcohol and reduces peak blood alcohol level and the area under the curve. Furthermore, carbohydrate meals may suppress the circulating and hepatic free fatty acid levels, thereby increasing alcohol metabolism (Thurman et al., 1972). The sugar in the product (fructose) increases alcohol metabolism by providing a substrate to help convert $\mathrm{NADH}$ to $\mathrm{NAD}^{+}$and to enhance mitochondrial oxygen uptake (Wissel, 1987; Passananti et al., 1990; Ramchandani et al., 2001). Lac- 


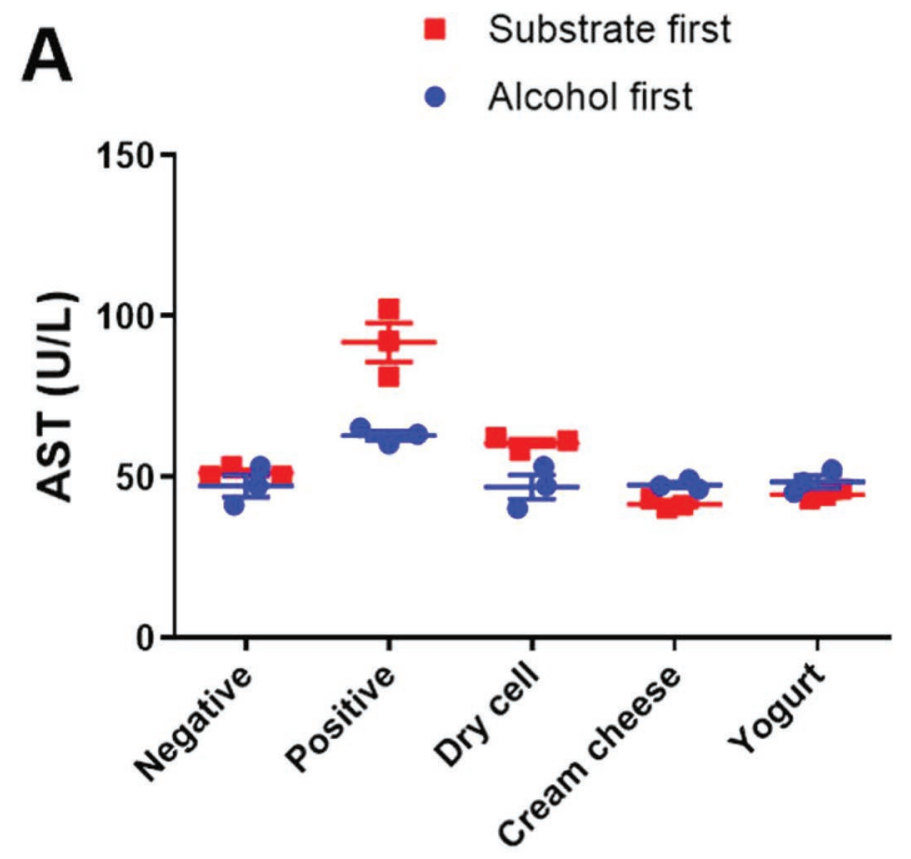

B

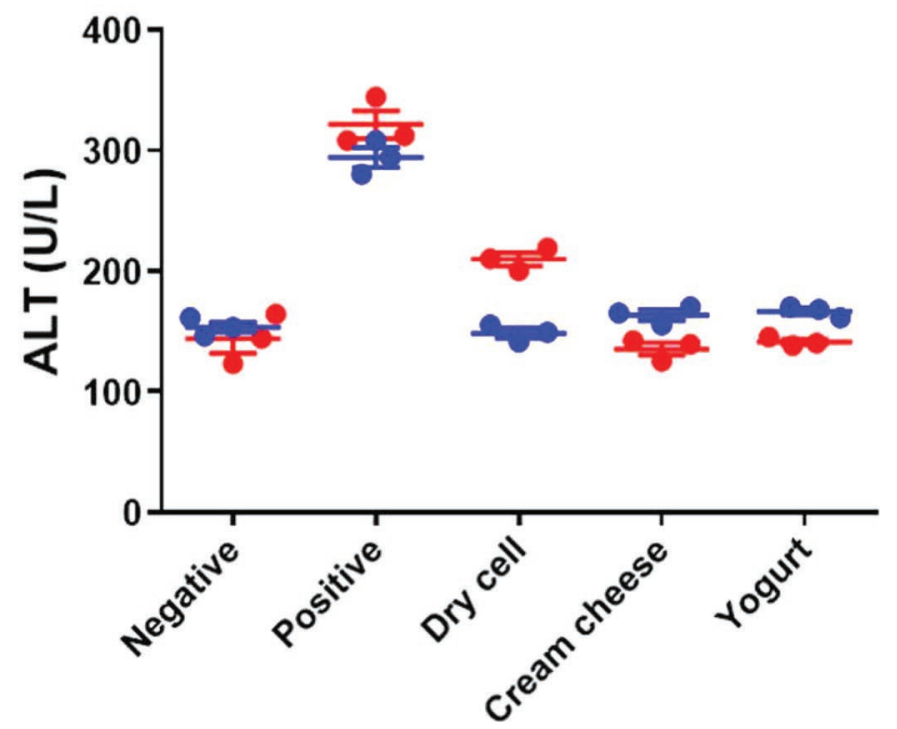

Figure 7. (A) Aspartate aminotransferase (AST), and (B) alanine aminotransferase (ALT) levels (in triplicate) in rat blood after treatment with $20 \%$ ethanol first and then Lactococcus lactis ssp. lactis ATCC 19435 in 1 of 3 substrates: freeze-dried cells, cream cheese, or yogurt; or after administration of Lc. lactis ssp. lactis ATCC in substrates first and then $20 \%$ ethanol. Negative = water only, Positive = $20 \%$ ethanol only. Data are shown as the mean \pm SEM. Color version available online.

tococcus chungangensis $\mathrm{CAU} 28$ produces both $\mathrm{ADH}$ and ALDH not only intracellularly but also in cream cheese and yogurt. Both of these enzymes have been effectively utilized for the regeneration of NADPH in multiple enzyme systems and have been used to reduce blood alcohol and acetaldehyde levels in rats. Many facultative anaerobic and aerobic gastrointestinal bacteria have exhibited high ADH (Jokelainen et al., 1996; Nosova et al., 1997) and catalase (Tillonen et al., 1998) activities and are therefore capable of producing acetaldehyde from ethanol in vitro under aerobic and microaerobic conditions (Salaspuro et al., 1999). Alcohol-treated rats (positive group) showed high activity of the toxicity markers AST and ALT, indicating increases in membrane permeability, cellular damage, and necrosis of hepatocytes (Baldi et al., 1993; Tahir and Sultana, 2011).

Acute alcohol intake markedly increased serum AST and ALT levels. However, administration of Lc. chungangensis substrates decreased serum AST and ALT levels in rats. Comparison of the 2 experiments - with substrate provided before or after administration of $20 \%$ ethanol-showed that ingestion of cream cheese both before and after treatment with $20 \%$ ethanol was optimal for reducing $\mathrm{ADH}$ and $\mathrm{ALDH}$ levels in the blood. These results suggested that Lc. chungangensis CAU 28 substrates could be used as functional foods to reduce hepatotoxicity by decreasing the levels of alcohol and acetaldehyde in the blood and levels of AST and ALT in serum. Our results support the protective effects of dairy products from Lc. chungangensis CAU 28 against alcohol-induced liver diseases, suggesting that these products could be used as nutraceuticals for the prevention and control of alcohol-induced damage.

\section{ACKNOWLEDGMENTS}

This work was supported by the National Research Foundation of Korea (NRF, Daejeon, Republic of Korea) grant funded by the Korea government (MSIP; NRF-2016R1A2B4008481).

\section{REFERENCES}

Agerholm-Larsen, L., A. Raben, N. Haulrik, A. Hansen, M. Manders, and A. Astrup. 2000. Effect of 8 week intake of probiotic milk products on risk factors for cardiovascular diseases. Eur. J. Clin. Nutr. 54:288-297.

Baldi, E., P. Burra, M. Plebani, and M. Salvagnini. 1993. Serum malondialdehyde and mitochondrial aspartate aminotransferase activity as markers of chronic alcohol intake and alcoholic liver disease. Ital. J. Gastroenterol. 25:429-432.

Brown, A. C., and A. Valiere. 2004. Probiotics and medical nutrition therapy. Nutr. Clin. Care 7:56-68.

Choi, W. J., M. Konkit, Y. Kim, M.-K. Kim, and W. Kim. 2016. Oral administration of Lactococcus chungangensis inhibits 2,4-dinitrochlorobenzene-induced atopic-like dermatitis in NC/Nga mice. J. Dairy Sci. 99:6889-6901.

Corrao, G., L. Rubbiati, V. Bagnardi, A. Zambon, and K. Poikolainen. 2000. Alcohol and coronary heart disease: A meta-analysis. Addiction 95:1505-1523.

Gerritse, K., M. Posno, M. Schellekens, W. Boersma, and E. Claassen. 1990. Oral administration of TNP-Lactobacillus conjugates in mice: A model for evaluation of mucosal and systemic immune 
responses and memory formation elicited by transformed lactobacilli. Res. Microbiol. 141:955-962.

Gomes, A. M., and F. X. Malcata. 1999. Bifidobacterium spp. and Lactobacillus acidophilus: Biological, biochemical, technological and therapeutical properties relevant for use as probiotics. Trends Food Sci. Technol. 10:139-157.

Gotcheva, V., E. Hristozova, T. Hristozova, M. Guo, Z. Roshkova, and A. Angelov. 2002. Assessment of potential probiotic properties of lactic acid bacteria and yeast strains. Food Biotechnol. 16:211-225.

Imase, K., A. Tanaka, K. Tokunaga, H. Sugano, H. Ishida, and S. Takahashi. 2007. Lactobacillus reuteri tablets suppress Helicobacter pylori infection-A double-blind randomised placebo-controlled cross-over clinical study. Kansenshogaku Zasshi 81:387-393.

Jokelainen, K., A. Siitonen, H. Jousimies-Somer, T. Nosova, R. Heine, and M. Salaspuro. 1996. In vitro alcohol dehydrogenase-mediated acetaldehyde production by aerobic bacteria representing the normal colonic flora in man. Alcohol. Clin. Exp. Res. 20:967-972.

Kalliomäki, M., S. Salminen, H. Arvilommi, P. Kero, P. Koskinen, and E. Isolauri. 2001. Probiotics in primary prevention of atopic disease: A randomised placebo-controlled trial. Lancet 357:10761079 .

Kimoto, H., J. Kurisaki, N. Tsuji, S. Ohmomo, and T. Okamoto. 1999 Lactococci as probiotic strains: Adhesion to human enterocyte-like Caco-2 cells and tolerance to low $\mathrm{pH}$ and bile. Lett. Appl. Microbiol. 29:313-316.

Konkit, M., W. J. Choi, and W. Kim. 2015. Alcohol dehydrogenase activity in Lactococcus chungangensis: Application in cream cheese to moderate alcohol uptake. J. Dairy Sci. 98:5974-5982.

Konkit, M., W. J. Choi, and W. Kim. 2016. Aldehyde dehydrogenase activity in Lactococcus chungangensis: Application in cream cheese to reduce aldehyde in alcohol metabolism. J. Dairy Sci. 99:1755-1761.

Konkit, M., J.-H. Kim, N. Bora, and W. Kim. 2014. Transcriptomic analysis of Lactococcus chungangensis sp. nov. and its potential in cheese making. J. Dairy Sci. 97:7363-7372.

Kurkivuori, J., V. Salaspuro, P. Kaihovaara, K. Kari, R. Rautemaa, L. Grönroos, J. H. Meurman, and M. Salaspuro. 2007. Acetaldehyde production from ethanol by oral streptococci. Oral Oncol. 43:181-186.

Lamed, R. J., and J. Zeikus. 1981. Novel NADP-linked alcohol-aldehyde/ketone oxidoreductase in thermophilic ethanologenic bacteria. Biochem. J. 195:183-190.

Lees, G. J., and G. Jago. 1976. Acetaldehyde: an intermediate in the formation of ethanol from glucose by lactic acid bacteria. J. Dairy Res. 43:63-73.

Maher, J. J. 1997. Exploring alcohol's effects on liver function. Alcohol Health Res. World 21:5-12.

Nanji, A. A., U. Khettry, and S. H. Sadrzadeh. 1994. Lactobacillus feeding reduces endotoxemia and severity of experimental alcoholic liver (disease). Proc. Soc. Exp. Biol. Med. 205:243-247.

Nomoto, K. 2005. Prevention of infections by probiotics. J. Biosci. Bioeng. 100:583-592.

Nosova, T., H. Jousimies-Somer, K. Jokelainen, R. Heine, and M. Salaspuro. 2000. Acetaldehyde production and metabolism by human indigenous and probiotic Lactobacillus and Bifidobacterium strains. Alcohol Alcohol. 35:561-568.

Nosova, T., H. Jousimies-Somer, P. Kaihovaara, K. Jokelainen, R. Heine, and M. Salaspuro. 1997. Characteristics of alcohol dehydrogenases of certain aerobic bacteria representing human colonic flora. Alcohol. Clin. Exp. Res. 21:489-494.

Passananti, G. T., C. A. Wolff, and E. S. Vesell. 1990. Reproducibility of individual rates of ethanol metabolism in fasting subjects. Clin. Pharmacol. Ther. 47:389-396.

Pearl, R. 1926. Alcohol and Longevity. Alcohol and Longevity, Knopf, New York.

Ramchandani, V. A., P. Y. Kwo, and T. K. Li. 2001. Effect of food and food composition on alcohol elimination rates in healthy men and women. J. Clin. Pharmacol. 41:1345-1350.
Rehm, J., R. Room, M. Monteiro, G. Gmel, K. Graham, N. Rehn, C. T. Sempos, and D. Jernigan. 2003. Alcohol as a risk factor for global burden of disease. Eur. Addict. Res. 9:157-164.

Rehm, J. T., S. J. Bondy, C. T. Sempos, and C. V. Vuong. 1997. Alcohol consumption and coronary heart disease morbidity and mortality. Am. J. Epidemiol. 146:495-501.

Rogers, J., J. Smith, G. Starmer, and J. Whitfield. 1987. Differing effects of carbohydrate, fat and protein on the rate of ethanol metabolism. Alcohol Alcohol. 22:345-353.

Room, R., T. Babor, and J. Rehm. 2005. Alcohol and public health. Lancet 365:519-530.

Room, R., D. Jernigan, B. Carlini-Marlatt, O. Gureje, K. Mäkelä, M. Marshall, M. E. Medina-Mora, M. Monteiro, C. Parry, and J. Partanen. 2002. Alcohol in Developing Societies: A Public Health Approach. Finnish Foundation for Alcohol Studies, Helsinki, Finland.

Salaspuro, V., S. Nyfors, R. Heine, A. Siitonen, M. Salaspuro, and H. Jousimies-Somer. 1999. Ethanol oxidation and acetaldehyde production in vitro by human intestinal strains of Escherichia coli under aerobic, microaerobic, and anaerobic conditions. Scand. J. Gastroenterol. 34:967-973.

Schrezenmeir, J., and M. de Vrese. 2001. Probiotics, prebiotics, and synbiotics-Approaching a definition. Am. J. Clin. Nutr. 73:361S$364 \mathrm{~S}$

Segawa, S., A. Hayashi, Y. Nakakita, H. Kaneda, J. Watari, and H. Yasui. 2008. Oral administration of heat-killed Lactobacillus brevis SBC8803 ameliorates the development of dermatitis and inhibits immunoglobulin e production in atopic dermatitis model NC/Nga mice. Biol. Pharm. Bull. 31:884-889.

Sehrawat, A., and S. Sultana. 2006. Evaluation of possible mechanisms of protective role of Tamarix gallica against DEN initiated and 2-AAF promoted hepatocarcinogenesis in male Wistar rats. Life Sci. 79:1456-1465.

Setshedi, M., J. R. Wands, and S. M. de la Monte. 2010. Acetaldehyde adducts in alcoholic liver disease. Oxid. Med. Cell. Longev. 3:178-185.

Shah, N. P. 2007. Functional cultures and health benefits. Int. Dairy J. 17:1262-1277.

Smith-Warner, S. A., D. Spiegelman, S.-S. Yaun, P. A. van den Brandt, A. R. Folsom, R. A. Goldbohm, S. Graham, L. Holmberg, G. R. Howe, and J. R. Marshall. 1998. Alcohol and breast cancer in women: A pooled analysis of cohort studies. JAMA 279:535-540.

Tahir, M., and S. Sultana. 2011. Chrysin modulates ethanol metabolism in Wistar rats: A promising role against organ toxicities. Alcohol Alcohol. 46:383-392.

Takada, A., M. Tsutsumi, M. Okudaira, Y. Ohta, T. Tsujii, K. Tanikawa, Y. Hasumura, N. Sato, H. Ishii, S. Harada, T. Okanoue, C. Sato, and S. Takase. 1995. National survey of alcoholic liverdisease in Japan (1968-91). J. Gastroenterol. Hepatol. 10:509-516.

Thurman, R. G., H. G. Ley, and R. Scholz. 1972. Hepatic microsomal ethanol oxidation. Eur. J. Biochem. 25:420-430.

Tillonen, J., P. Kaihovaara, H. Jousimies-Somer, R. Heine, and M. Salaspuro. 1998. Role of catalase in in vitro acetaldehyde formation by human colonic contents. Alcohol. Clin. Exp. Res. 22:1113-1119.

Vanni, A., E. Pessione, L. Anfossi, C. Baggiani, M. Cavaletto, M. Gulmini, and C. Giunta. 2000. Properties of a cobalt-reactivated form of yeast alcohol dehydrogenase. J. Mol. Catal. B Enzym. 9:283-291.

Voigt, M. D. 2005. Alcohol in hepatocellular cancer. Clin. Liver Dis. 9:151-169.

Walsh, K., and G. Alexander. 2000. Alcoholic liver disease. Postgrad. Med. J. 76:280-286.

Wissel, P. S. 1987. Dietary influences on ethanol metabolism. Drug Nutr. Interact. 5:161-168 